\title{
Método de decisão dos processos de prototipagem rápida na concepção de novos produtos
}

\section{Decision method for rapid prototyping processes in new products design}

\author{
Osiris Canciglieri Junior ${ }^{1}$ \\ Aguilar Selhorst Junior ${ }^{1}$ \\ Ângelo Márcio Oliveira Sant'Anna²
}

\begin{abstract}
Resumo: A tecnologia de prototipagem de produtos por adição de material (Rapid Prototyping) e por remoção de material (Subtractive Rapid Prototyping) pode variar em: qualidade, tempo e custos, dependendo das características do produto. A escolha da tecnologia muitas vezes está condicionada à decisão empírica do designer/engenheiro ou operador, devido ao tipo de material, dimensão e precisão. Este artigo apresenta um método para tomada de decisão que auxilia a escolha da tecnologia de prototipagem rápida mais adequada na concepção de novos produtos. Foram utilizados os conceitos de DFM (Design for Manufacturing), CAD (Computer Aided Design), RPD (Rapid Product Development) e MAUT (Multiattribute Utility) em dois produtos de diferente estrutura e material (composto leve e simples maciço) para avaliar e validar o método proposto. Os resultados apresentam comparações entre as tecnologias de prototipagem rápida a partir de critérios para tomada de decisão, aperfeiçoando a concepção de protótipos mais eficientes.
\end{abstract}

Palavras-chave: Engenharia do produto; Prototipagem rápida; Tomada de decisão; Critério multiatributos.

\begin{abstract}
The prototyping of products by additive material (Rapid Prototyping) and by subtractive material (Subtractive Rapid Prototyping) technology can vary in: quality, time and costs, depending on the product characteristics. The selection of technology often is conditioned to the designer/engineer or operator empirical decision due to the type of material, size and accuracy. This paper presents a method for decision making to assist the best choice of rapid prototyping technology on new product conception. We used the concepts of design for manufacturing (DFM), rapid product development $(R P D)$ and computer aided design $(C A D)$ for two products of different structure and material (lightweight dual and simple solid) to evaluate and validate the proposed method. The results show comparisons between the rapid prototyping technologies based on decision making, improving the conception of most efficient prototype.
\end{abstract}

Keywords: Product engineering; Rapid prototyping; Decision making; Multiattribute criteria.

\section{Introdução}

Atualmente a informação, o conhecimento e a capacidade de resposta às necessidades do mercado são ativos valiosos que as empresas utilizam para se tornarem competitivas em um mercado que exige uma grande variedade de produtos de qualidade com tempo e custo reduzido. Para isso, as empresas estão utilizando as novas tecnologias de informação para integrar vários setores das organizações por meio do conhecimento dos indivíduos e de tecnologias e equipamentos, capazes de promover e interpretar os resultados desta integração.
A integração e a rapidez entre as várias fases do desenvolvimento de produtos são sem dúvida elementos-chave na competitividade entre as empresas. Assim, a passagem rápida do conceito do produto à produção é um incremento de competitividade que a prototipagem por meio de sistemas CAD/CAM (Computer Aided Design/Manufacture) oferece às empresas como meio determinante para o sucesso do desenvolvimento de novos produtos (Porto et al., 2002).

As indústrias de produtos de ciclos rápidos (ciclo de vida por dois anos ou menos) utilizam algumas estratégias que reduzem o tempo de desenvolvimento

\footnotetext{
${ }^{1}$ Programa de Pós-graduação em Engenharia de Produção e Sistemas, Pontifícia Universidade Católica do Paraná - PUCPR, Rua Imaculada Conceição, 1155, CEP 80215-901, Curitiba, PR, Brasil, e-mail: osiris.canciglieri@ pucpr.br; aguilar.junior@pucpr.br

${ }^{2}$ Departamento de Engenharia de Produção, Universidade de São Paulo - USP, Rua Prof. Luciano Gualberto, 308, CEP 05508-010, São Paulo, SP, Brasil, e-mail: angeloms@usp.br
}

Recebido em Abr. 11, 2013 - Aceito em Fev. 05, 2015

Suporte financeiro: Pontífica Universidade Católica do Paraná. 
de produtos: $(i)$ escutando a voz do consumidor para entender suas necessidades logo no início do ciclo de desenvolvimento; (ii) investindo em capacitação técnica para rápida aprendizagem e traduzindo as necessidades dos consumidores em desenvolvimento de novos produtos; (iii) desenvolvendo grupos que interagem interdisciplinarmente para engenharia concorrente; e (iv) promovendo aprendizado continuo através de rápidas modificações nos produtos e na extensão das linhas de produto (Evans \& Campbell, 2003).

Na busca pela integração dos vários setores das organizações industriais e comerciais, a prototipagem rápida pode ser o elemento aglutinante dos diferentes interesses desses setores, pois a rapidez e consolidação dessa integração é elemento-chave na competitividade entre as empresas. Uma vez estabelecido o design do produto, que engloba aplicações, usuários, processos e especificações, o projeto torna-se necessário, onde tal conceito será desenvolvido até encontrar-se pronto para a produção. Essa sequência estabelece o desenvolvimento de um produto, e esse desenvolvimento só terá seus riscos minimizados a partir do momento em que os elementos que o compõem forem examinados por aqueles que fazem parte do processo de desenvolvimento (Campbell et al., 2007). Em determinados momentos do processo de desenvolvimento, modelos e protótipos são fundamentais para tais exames. Ao finalizar o conceito de um produto, por exemplo, a análise volumétrica permite uma visão análise formal, ergonômica e de usabilidade para estabelecer o design do produto; neste ponto já é possível perceber os valores simbólicos ou alegóricos que o produto representará (Ferreira et al., 2006).

A construção de protótipos rápidos é de elevada importância, pois fisicamente, e não apenas em papel ou imagens virtuais, é possível obter certo grau de interação com o produto ou seu volume. Analisando as execuções de protótipos rápidos em tecnologias de prototipagem, observa-se que os protótipos são gerados em questão de horas e não de dias ou semanas, como era antes do surgimento destas tecnologias de construção de protótipos. Dessa forma, atualmente é notória a difusão da prototipagem rápida envolvendo diversas áreas do conhecimento, tais como: a engenharia de produto; a área médica na concepção de implantes (Sun et al., 2013); as engenharias mecânica e elétrica no desenvolvimento de produtos como pilha a combustível (Jain et al., 2012).

O uso das tecnologias de prototipagem rápida está relacionado ao propósito do protótipo, a flexibilidade dos materiais e o tempo de execução. Há tecnologia que não atende todas as demandas de análise de um protótipo, mas consegue atender às demandas mais relevantes de um protótipo, inclusive considerar elementos relacionados à redução de risco ou requisitos sustentáveis (Canciglieri et al., 2014).
Nesse contexto, este artigo apresenta um método para tomada de decisão que auxilia a escolha da tecnologia de prototipagem rápida mais adequada na concepção de novos produtos. O intuito é fornecer uma ferramenta de suporte na tomada de decisão entre as tecnologias de prototipagem rápida por adição e remoção de materiais, fundamentada nos conceitos de DFM (Design for Manufacturing), CAD (Computer Aided Design), RPD (Rapid Product Development) e MAUT (Multiattribute Utility).

Na seção 2, é apresentada uma breve revisão das tecnologias de prototipagem rápida. A seção 3 apresenta o método de tomada de decisão proposto. Na seção 4, uma aplicação do método proposto por meio de um estudo experimental. Finalmente, na seção 5, as conclusões e perspectivas futuras.

\section{Prototipagem rápida}

Rapid Product Development (RPD) é um processo iterativo que envolve manufatura e a evolução de protótipos rápidos, caracterizado por equipes de design distintas cooperando entre si de forma paralela. Na prática, o processo de RPD é reflexo de uma competição pela melhor solução em um grupo de alternativas de soluções. A combinação deste grupo de alternativas, seu desenvolvimento e suas adaptações em um ambiente de troca dinâmico entre as equipes de design é que irão gerar produtos colocados no mercado com mais qualidade, menos tempo e custo de desenvolvimento (Volpato, 2007). Em contrapartida ao crescimento da $\mathrm{RP}$, novas pesquisas ao processo de usinagem trouxeram ao mercado equipamentos CNC de bancada com custos mais atraentes do que aqueles praticados nos tradicionais centros de usinagem, mostrando-se uma alternativa à RP tradicional, fazendo assim surgir o conceito de SRP (Subtrative Rapid Prototyping).

\subsection{SRP: subtração de material}

O processo SRP (Subtractive Rapid Prototyping) é comumente confundido com o VMC (Vertical Machining Center), os centros de usinagem, pelas semelhanças dos equipamentos. Na verdade, a base da tecnologia é a mesma, ambos iniciam o processo de fabricação a partir de um bloco de material, desbastando-o até obter o objeto desejado. Ambos desbastam uma variedade enorme de materiais, desde metais aos mais usuais dos polímeros como o nylon, ABS, Poliacetal, Acrílicos, etc. Utilizam-se por décadas de desenvolvimento tecnológico em controles de precisão dos eixos de usinagem com servos-motores AC, trocadores de ferramentas, eixos adicionais de usinagem, entre outros. Entretanto, existem diferenças significativas entre o processo de SRP e os centros de usinagem (VMCs). Enquanto os VMCs são otimizados para a confecções de objetos metálicos e ferramental 
em aço, os equipamentos de SRP são equipamentos pequenos, com perfil de desktops, cabendo em escritórios de desenvolvimento, podendo ser ligados em redes domésticas de energia elétrica e necessitam de pouca manutenção. Trabalham com baixo nível de ruído e não utilizam sistemas de refrigeração e filtragem. São fáceis de operar e geralmente contam com software CAM integrado e otimizado para o processo de prototipagem, proporcionando a designers e engenheiros a possibilidade de criação de protótipos rápidos de excelente precisão e acabamento superficial (Wohlers \& Grimm, 2003).

As propriedades mecânicas dos protótipos ficam por conta dos materiais a serem usinados, que além daqueles polímeros usuais, contam como grande variedade de poliuretanos que cada vez mais simulam os polímeros pretendidos na produção em escala do protótipo desenvolvido. A utilização de metais não ferrosos também é usual nos processos de SRP.

O acabamento superficial e o tempo de execução dos protótipos também sofrem influência direta do tipo de material, das ferramentas de usinagem e dos controles via software das atividades do equipamento antes da construção do objeto. Assim, a qualidade do acabamento superficial está sujeita ao objetivo final do protótipo e não apenas à qualidade que o equipamento oferece. Outra possibilidade em alguns equipamentos de SRP é a troca do cabeçote de usinagem por scanner tridimensional, capaz de fazer a leitura de objetos muitas vezes prototipados manualmente e que servem de base para o desenho CAD, onde se viabilizará a correção de imperfeições de superfícies, para então a usinagem posterior, no mesmo equipamento, de um protótipo mais próximo do pretendido. Portanto, a diversidade de materiais e a flexibilidade do processo de SRP, além da velocidade de resposta à execução de protótipos, habilitam este processo ao conceito de prototipagem rápida.

\subsection{RP: adição de material}

Os mais entusiastas das tecnologias de RP (Rapid Prototyping) acreditam que no futuro os equipamentos serão caseiros, quando será possível utilizá-los como uma impressora de papel comum a todos os desktops ao redor do mundo. A tecnologia de RP conta com processos novos a cada ano, embora em algumas empresas ainda sejam utilizados processos estabelecidos há mais de quinze anos (Warfel, 2002). Porém, existe ainda uma distância considerável a ser vencida quanto aos custos dos equipamentos e quanto ao desenvolvimento de outras tecnologias que vêm se mostrando eficientes na construção de protótipos e que não usam o conceito básico de adição de materiais em camadas (Newcomer et al., 2004). Existem disponíveis ao mercado hoje diversos equipamentos que proporcionam a construção de protótipos rápidos via adição de camadas. Todos esses equipamentos têm em comum a possibilidade de construção de geometrias complexas, sem a necessidade da utilização de qualquer tipo de ferramenta de construção do tipo moldes, ou seja, constroem-se os objetos de forma livre, o que fez surgir recentemente a denominação de Solid Freeform Fabrication - fabricação de sólidos de forma livre, pois o termo tradicional Rapid Prototyping - Prototipagem Rápida vem se tornando mais usual e correto a todo tipo de prototipagem de rápida execução, independentemente do processo de adição ou remoção de material, ou ainda independentemente do uso ou não das tecnologias que envolvem hardwares e softwares. Já que um protótipo rápido feito em massa de modelar pode efetivamente apresentar elementos suficientes para a decisão de ir em frente ou não com uma alternativa de projeto de determinado produto. A tecnologia de RP por adição oferece ao mercado processos como:

- LOM (Laminated Object Manufacturing) - com base em folhas de papel, oferece baixo custo;

- FDM (Fused Deposition Modeling) - com filamento em ABS (acrilonitrila butadieno estireno), proporcionando durabilidade e resistência;

- SLA (StereoLihography Apparatus) - oferecendo translucidez e precisão;

- SLS (Selective Laser Sintering) - com base de material em nylon (poliamida) ou metal, oferecendo resistência mecânica e resistência ao calor;

- 3Dprint (Three-dimensional Printing) - com base de camada em pó e adesivo líquido, oferecendo rapidez e visualização.

Os esforços das indústrias de equipamentos de prototipagem rápida são imensos para tentar resolver questões da representação de todos os aspectos possíveis em produtos poliméricos, metálicos, cerâmicos ou compostos. Os equipamentos de SLS são capazes de sintetizar camadas de poliamida com vidro, poliamida com metais e metais não ferrosos. Equipamentos de FDM constroem protótipos com filamentos de ABS, policarbonato, a combinação deles e outros polímeros compostos. Equipamentos de SLA constroem protótipos a partir de resinas que simulam o ABS e Polipropileno. E os equipamentos de 3DPrint são capazes de construir protótipos a partir de gesso com partes coloridas e com propriedades flexíveis. A Tabela 1 resume algumas características das tecnologias de RP disponíveis no país. 
Tabela 1. Características da prototipagem rápida no Brasil.

\begin{tabular}{|c|c|c|c|c|c|}
\hline \multirow{2}{*}{ Fatores Determinantes } & \multicolumn{5}{|c|}{ Processos } \\
\hline & LOM & FDM & SLA & SLS & 3DPrint \\
\hline Variedade de Materiais & Pequena & Média & Pequena & Grande & Média \\
\hline Translucidez & Não & Sim & Sim & Não & Não \\
\hline Qualidade Superficial & Regular & Regular & Regular & Boa & Boa \\
\hline Pós-Acabamento superficial & Baixa & Regular & Regular & Boa & Boa \\
\hline Precisão & Baixa & Regular & Excelente & Boa & Boa \\
\hline Resistência ao Impacto & Baixa & Boa & Regular & Boa & Baixa \\
\hline Resistência a Flexão & Baixa & Excelente & Baixa & Excelente & Baixa \\
\hline Custo do Protótipo no Brasil & Alto & Médio & Alto & Médio & Médio \\
\hline Pós-Processo & Sim & Sim & Sim & Sim & Sim \\
\hline Pós-Cura & Não & Não & Sim & Não & Não \\
\hline
\end{tabular}

\section{Método de auxílio à tomada de decisão}

Ao se comparar as características e tecnologias de prototipagem rápida por remoção (SRP) e por adição (RP), é necessário compreender quais são as limitações de cada tecnologia e processo. Este estudo avaliou as características existentes na prototipagem rápida, a fim de definir as características relevantes que proporcionaram a base para os critérios de decisão das tecnologias de prototipagem rápida. A seleção da melhor tecnologia geralmente está associada ao tempo disponível para execução do protótipo, entretanto, deve-se analisar o propósito e o custo no desenvolvimento do produto. Selecionar qual tecnologia utilizar para construir um determinado protótipo é mais abrangente do que uma simples opinião a respeito, ou seja, requer o conhecimento das tecnologias disponíveis e critérios de decisão para que se possa escolher a alternativa mais adequada. Segundo Borille et al. (2010), o potencial da tecnologia é obtido quando se define a priori relevantes elementos do produto a ser prototipado, tais como: precisão, acurácia, funcionalidade e custo. Conforme Machado \& Sant'Anna (2013), ao avaliar elementos das peças ou produtos em desenvolvimento, pode-se definir quais tecnologias atendem ou não aos requisitos exigidos do projeto para o desenvolvimento do produto (protótipo). Sant'Anna (2015) afirma que, a escolha de alternativas baseada em métodos de apoio à deisão tende a ser mais relevante para os usuários, uma vez que estes apresentam menor complexidade e maior acurácia do que os métodos analíticos.

Dessa forma, conhecendo a priori as características das tecnologias e de utilização do protótipo, é possível escolher a prototipagem rápida a ser usada como ponto de partida na tomada de decisão. O método de tomada de decisão proposto está dividido em 3 fases: (i) Descrição das características e geometria do produto; (ii) Aplicação dos critérios de decisão; (iii) Análise de tomada de decisão e (iii) Prototipagem do produto (ver Figura 1).

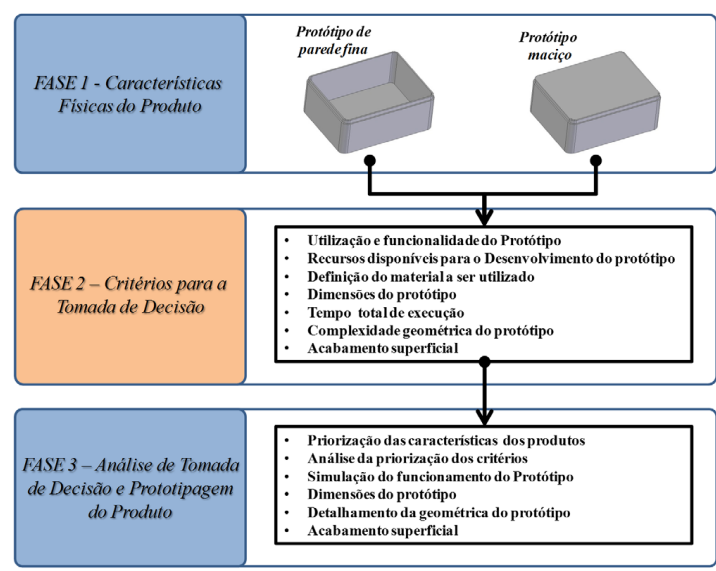

Figura 1. Método de tomada de decisão proposto.

\subsection{Fase 1: descrição das características do produto}

Nesta fase analisa-se o produto que será prototipado, considerando os aspectos dimensionais, geometria, características e restrições com base no propósito de utilização, a fim de definir a posteriori quais destas são mais relevantes para a tomada de decisão.

As principais características (critérios) relevantes a serem consideradas no método proposto foram definidas a partir da intensa revisão da literatura e feedback de especialistas em prototipagem, descritas a seguir:

1) Propósito: é um aspecto que define precisamente para que o protótipo será construído. Por exemplo, um protótipo que será exaustivamente testado deve ser considerado de maneira diferente de um protótipo que receberá avaliação estética, pois as tecnologias e processos de prototipagem rápida existentes no mercado possuem acabamentos superficiais diferentes. 
2) Material: é um aspecto necessário que define quais materiais podem ser utilizados para a confecção do protótipo, pois cada tecnologia de prototipagem conta com uma gama específica de materiais que pode utilizar como substrato na construção do protótipo. Por exemplo, a SRP, que pode usar uma infinidade de materiais, conta com o problema de disponibilidade de materiais poliméricos para usinagem.

3) Dimensão: é um aspecto que define as dimensões do protótipo a ser desenvolvido. As dimensões da geometria são pontos importantes para a definição de qual tecnologia utilizar e como utilizá-la, pois cada equipamento que pode ser usado para prototipagem rápida tem área de trabalho definida. Ademais, o tamanho do protótipo (volume) tem impacto direto no tempo de execução do protótipo e no custo.

4) Custos: é um aspecto fundamental para a equipe de desenvolvimento ter em mãos para a confecção do protótipo. Este item deve especificar o custo que cada peça-protótipo representa, de forma que seja possível comparar os custos das peças entre as tecnologias e processos de prototipagem rápida.

5) Tempo: é um aspecto que define o tempo necessário para execução de cada protótipo em cada tecnologia, incluindo-se o tempo de setup, execução dos protótipos e o pós-acabamento das peças.

6) Detalhamento: é um aspecto que define com precisão os detalhes da complexidade da geometria do protótipo. Deve-se avaliar se cada tecnologia e processo de prototipagem rápida são capazes de reproduzir com precisão os detalhes geométricos existentes nos protótipos, e se estes estão de acordo com os requisitos de projeto. Ademais, deve-se considerar o quanto o protótipo pode fugir das dimensões especificadas em projeto.

7) Acabamento Superficial: é um aspecto que está relacionado às informações referentes ao propósito do protótipo. No caso de um protótipo de apresentação estética, o acabamento superficial é importantíssimo, pois é ele que definirá o futuro do projeto; já para o caso de um protótipo funcional, nem sempre o acabamento superficial é fundamental.

\subsection{Fase 2: critérios para a tomada de decisão}

Nesta fase avaliam-se criteriosamente as características mais relevantes do produto e das tecnologias de prototipagem disponíveis para concepção do protótipo. A composição destes "critérios de decisão" visa definir o grau de prioridade para as características apresentadas na Fase 1 do método. Dessa forma, permite estabelecer os pesos dos critérios de priorização das características; a priorização e o ranqueamento das características mais relevantes.

Os pesos que definem o grau de importância que a característica analisada tem em relação ao produto e à tecnologia podem ser atribuídos por um grupo de especialistas (designer ou engenheiro ou operador da tecnologia). A intensidade dos pesos é definida por uma escala discreta de 0 a 9 , com o valor 0 indicando nenhuma importância e 9 extrema importância.

A escala dos pesos atribuídos foi baseada nos conceitos simples de modelagem de preferência da teoria de avaliação multiatributos (Multiattribute Utility Function-MAUT), comumente utilizada para atribuir prioridade aos critérios por meio de cálculos simples e eficazes (Min, 1994). O método MAUT permite representar as preferências do avaliador por probabilidades marginais dos atributos com base nos seus pontos de vista (critérios). A modelagem de preferência é realizada por meio da função utilidade (Miranda \& Almeida, 2004). Hinloopen et al. (2004) aplicam o método multiatributo MAUT para determinar a importância dos critérios de julgamento de dez sistemas de transporte público em uma cidade, com base na opinião de especialistas.

Um grupo de especialistas deve atribuir os pesos e as características (critérios), em uma escala numérica de 0 a 100, sendo 0 valor de importância mínima e 100 a importância máxima $\left(p a_{j}, j=1, \ldots, n\right)$. A próxima etapa consiste em determinar a priorização dos critérios das tecnologias de prototipagem identificadas, expressa pelo indicador $p t_{i j}, i=1, \ldots, m$, bem como os critérios de importância para o cliente $\left(p c_{i}, i=1, \ldots, m\right)$. Este indicador $Q_{j}$ deve ser estabelecido pelo feedback das características relevantes para o cliente. Assim, o resultado final das características priorizadas pode ser obtida pela Equação 1:

$$
Q_{j}=\sum_{i=1}^{m}\left(p a_{i} \times p t_{i j}\right) \times p c_{j}
$$

onde: $Q_{j}$ é o resultado associado ao valor ponderado de priorização das $i$ características do produto para cada $j$ características das tecnologias de prototipagem. O resultado esperado pela relação entre a importância dos atributos do produto e as características relevantes da tecnologia permite quantificar os critérios mais importantes da interação produto $x$ tecnologia, embora a inclusão dos critérios de importância dada pelo 
cliente reflete a priorização ideal das características relevantes.

A etapa final consiste em ranquear os valores obtidos do indicador $Q_{j}$, definindo as características priorizadas e mais relevantes. Ao analisar as importâncias das características com esses critérios de decisão e o conhecimento das tecnologias, as equipes de desenvolvimento podem definir quais tecnologias de prototipagem rápida serão mais adequadas para concepção dos protótipos em diversas geometrias de produto.

\subsection{Fase 3: análise de tomada de decisão e prototipagem do produto}

Nesta fase analisa-se comparativamente cada tecnologia de prototipagem disponível para concepção do protótipo, com base nos resultados obtidos na Fase 2 do método proposto, proporcionando um auxílio na tomada de decisão. Dessa forma, o resultado desta fase será a concepção do protótipo fundamentada nos critérios de decisão, proporcionando confiabilidade do protótipo, economia de tempo, recursos técnicos e financeiros e precisão/acuracidade das dimensões e formas geométricas.

\section{Estudo experimental}

Para a aplicação método de auxílio à tomada de decisão proposto entre as tecnologias de prototipagem rápida, foram selecionados dois produtos. $\mathrm{O}$ primeiro é um protetor de cerdas para escovas dentais (produto composto leve) apresentado na Figura 2a, e o segundo é uma haste de escova dental (produto simples maciço) apresentado na Figura 2b. Esses

(a)

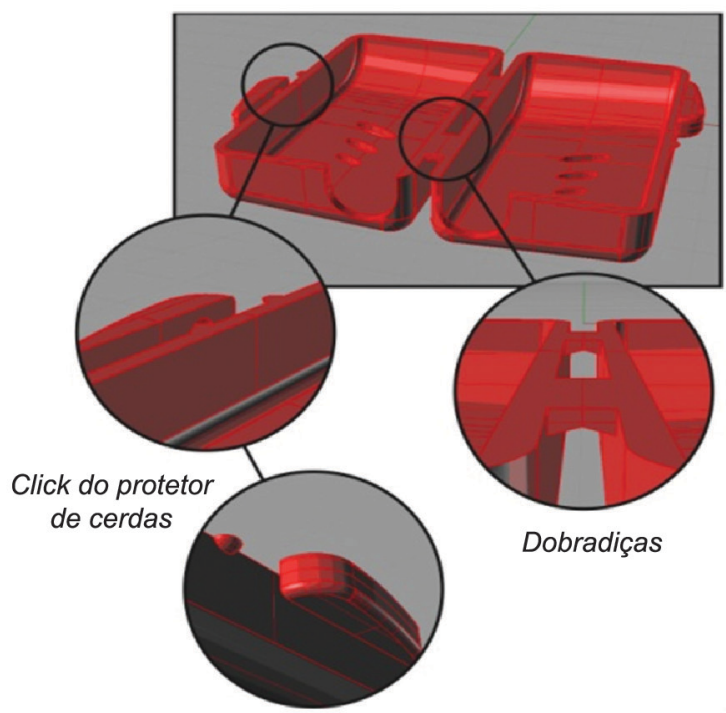

Figura 2. Produtos utilizados na aplicação do método proposto. produtos passaram pelo processo de criação, análise e modelagem tridimensional no sistema CAD. A escolha desses produtos teve por base a possibilidade de comparação entre os protótipos construídos e os produtos finais, pois estes produtos têm diferentes graus de complexidade, distintas geometrias, funções e massa, e são produzidos em materiais poliméricos distintos.

\subsection{Fase 1: análise das características do produto}

O primeiro produto é caracterizado por peças compostas de paredes finas. Nesta categoria, os produtos possuem elementos complexos como dobradiças e clicks de encaixe. O segundo produto é caracterizado por peças simples de corpo maciço, que possui relevos de pega e furações para encerdamento, que também possuem complexidade na sua formação geométrica.

Caso 1: O protetor de cerdas tem a função de proteger as cerdas de uma escova dental de contato com superfícies sujas e de pó (ver Figura 2a). Sua espessura média é de $1,00 \mathrm{~mm}$, sendo injetado em Polipropileno Random, para garantir a flexibilidade de duas dobradiças plásticas de $0,50 \mathrm{~mm}$ de espessura. Além disso, possui um sistema de fechamento com clicks, com $1,50 \mathrm{~mm}$ de diâmetro, que permite ao protetor ser fechado em torno das cerdas de escovas dentais. Estes clicks e dobradiças devem permitir a abertura e fechamento do protetor por pelo menos 3 meses de uso diário, conforme recomendação de projeto baseado em exigências do fabricante.

A dificuldade na prototipagem deste produto encontra-se na acuidade de sua espessura, tanto

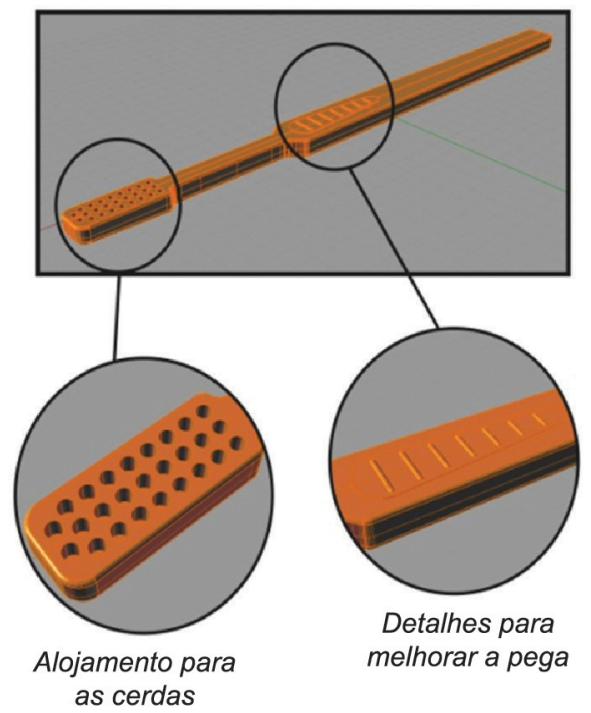


na casca do produto quanto na dobradiça plástica. Os clicks de fechamento formam em seu furo de fechamento um ângulo negativo para a extração do molde de injeção e, por consequência, na construção dos protótipos. Pode-se imaginar a dificuldade de prototipagem SRP (remoção), quando a usinagem executar este ângulo negativo específico da peça e um furo semicircular de $1,50 \mathrm{~mm}$ de diâmetro. Estas dimensões pequenas (dos furos dos clicks), bem como nas dobradiças plásticas, representam dificuldades de execução em alguns processos de RP (adição), pois algumas tecnologias de RP não conseguem reproduzir essas medidas.

Caso 2: A escova dental tem a função de realizar escovação dos dentes e possui a forma do corpo reta (ver Figura 2b), seguindo as recomendações da maioria dos dentistas. Foi projetada para suportar as pressões existentes no ato da escovação. Sua espessura média é de $5,50 \mathrm{~mm}$ e, por exigência de produção, pode ser injetada em Politereftalato de Etila (PET), ou em Polipropileno Random (PP Random), ou em Polietileno de Alta Densidade (PEAD), para garantir a flexibilidade na escovação e também a manutenção de sua forma reta durante a escovação. Ademais, possui detalhe de pega com relevos de $1,00 \mathrm{~mm}$ de diâmetro, permitindo que a escova não escorregue durante seu uso. Possui 28 furos de 1,80mm de diâmetro e 2,50mm de profundidade, que são utilizados para encravar as cerdas de Poliamida ou Nylon. Através de âncoras metálicas, as cerdas são colocadas uma a uma em sistema de encravamento automático, em menos de 2 segundos por escova.

A dificuldade na prototipagem desse produto encontra-se na acuidade de sua estrutura, na formação dos relevos de pega e principalmente na manutenção dos diâmetros dos furos de encerdamento, pois o produto deve ser compatível com o sistema de encravamento das cerdas. Tanto na prototipagem SRP (remoção) quanto na RP (adição), o desafio se dá tanto na usinagem correta dos diâmetros dos furos de encravamento das cerdas, quanto na formação dos relevos de pega. A Tabela 2 apresenta a descrição das características, componentes e funcionalidades dos produtos em estudo em consonância com os requisitos do cliente.

\subsection{Fase 2: aplicação dos critérios de decisão}

Uma pesquisa foi realizada com um grupo de especialistas formado por 5 pessoas, sendo 2 oriundos de Universidades e 3 de Indústrias da região (segmento eletrodomésticos, injetores e metal-mecânico), com objetivo de obter percepções e ponderações acerca do estudo. O grupo de especialistas opinou quanto aos seguintes aspectos: (i) peso para as características das tecnologias $\left(p a_{i}\right)$ e (ii) a priorização dos critérios
Tabela 2. Descrição das características dos produtos em estudo.

\begin{tabular}{|c|c|c|}
\hline Características & $\begin{array}{l}\text { Protetor de } \\
\text { Cerdas }\end{array}$ & Escova dental \\
\hline Propósito & $\begin{array}{c}\text { Teste de } \\
\text { funcionamento }\end{array}$ & $\begin{array}{c}\text { Teste de } \\
\text { funcionamento }\end{array}$ \\
\hline Material & Polipropileno & $\begin{array}{l}\text { Politereftalato de } \\
\text { etila (PET) }\end{array}$ \\
\hline Dimensão & $\begin{array}{c}47,70 \times 37,50 \times \\
8,90 \mathrm{~mm}\end{array}$ & $\begin{array}{c}180,00 \times 12,50 \times \\
8,90 \mathrm{~mm}\end{array}$ \\
\hline Custo & Alto & Alto \\
\hline Tempo & Horas & Horas \\
\hline Detalhamento & Detalhes precisos & Furos precisos \\
\hline $\begin{array}{l}\text { Acabamento } \\
\text { Sup. }\end{array}$ & $\begin{array}{c}\text { clicks e } \\
\text { dobradiças }\end{array}$ & Relevo de pega \\
\hline
\end{tabular}

relevantes do produto com relação às características das tecnologias $\left(p t_{i j}\right)$. A intensidade dos pesos (importância) dos critérios relevantes do produto foi definida a priori pelo cliente $(p c)$ e foram apresentados ao grupo após a finalização da atividade, a fim de evitar influência em suas opiniões.

Os pesos para as características das tecnologias de prototipagem foram atribuídos em um brainstorming realizado com os membros do grupo de especialistas com base em suas percepções e experiências (ver Tabela 3). Estes pesos $\left(p a_{i}\right)$ permitem indicar a importância de cada característica das tecnologias que são utilizadas no método.

Determinada a priorização das características das tecnologias de prototipagem, os grupos de especialistas atribuíram o grau de importância da relação entre as características do produto e das tecnologias, considerando o tipo do produto, permitindo construir a matriz de priorização das características e respectivas pontuações. As Tabelas 4 e 5 apresentam as matrizes de priorização das características das tecnologias e dos produtos 1 (protetor de cerdas) e produto 2 (escova dental), respectivamente. As Figuras 3 e 4 ilustram o ranqueamento das características relevantes dos produtos baseado na priorização das características das tecnologias SRP e RP dos produtos 1 e 2, respectivamente. Observa-se que as características (critérios) do produto mais relevantes são: propósito, material e detalhamento, embora haja diferença conforme o tipo do produto.

A aplicação do método de priorização multiatributo de decisão para os dois produtos em estudo forneceu resultados interessantes, destacando que os protótipos dos produtos devem ser construídos utilizando as tecnologias de prototipagem RP (adição de material) para o produto 1 (protetor de cerdas) com $Q_{1}=296 \mathrm{e} \mathrm{de}$ SRP (subtração de material) para o produto 2 (escova dental) com $Q_{1}=241$, conforme Tabelas 4 e 5 . 

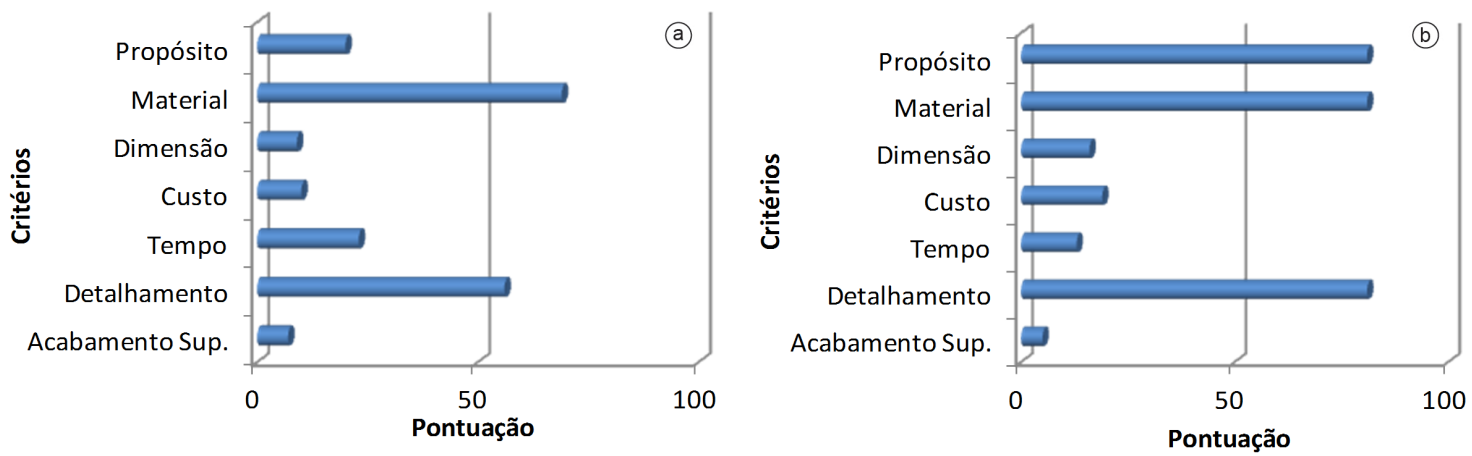

Figura 3. Ranqueamento dos critérios do produto 1 por tecnologia SRP (a) e RP (b).
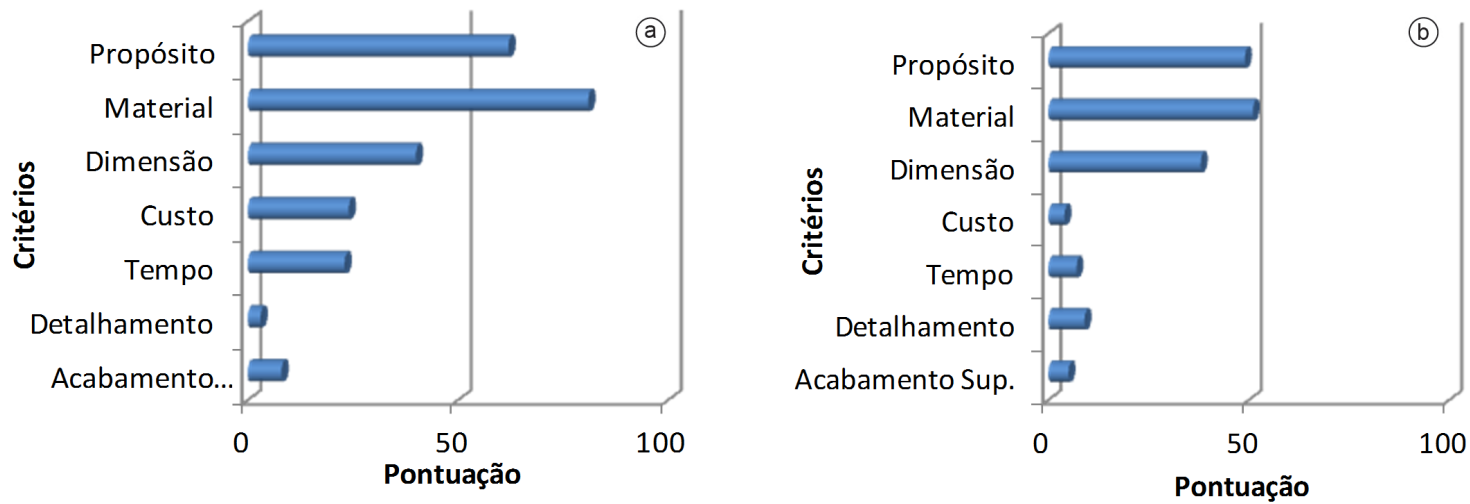

Figura 4. Ranqueamento dos critérios do produto 2 por tecnologia SRP (a) e RP (b).

Tabela 3. Peso atribuído às características das tecnologias.

\begin{tabular}{ccc}
\hline \multicolumn{1}{c}{ Características } & Peso & pa $_{i}$ \\
\hline Formas do processo & 40 & $24 \%$ \\
$\widetilde{\sim}$ Risco técnico & 60 & $35 \%$ \\
Complexidade & 40 & $24 \%$ \\
Inovação & 30 & $18 \%$ \\
Importância & 170 & $36 \%$ \\
Formas do processo & 50 & $17 \%$ \\
Risco técnico & 60 & $20 \%$ \\
Complexidade & 90 & $30 \%$ \\
Inovação & 100 & $33 \%$ \\
Importância & 300 & $64 \%$ \\
\hline
\end{tabular}

\subsection{Fase 3: análise de tomada de decisão e prototipagem do produto}

Com objetivo de validar o método de decisão proposto, foi realizada a prototipagem dos dois produtos em estudo, utilizando os tipos de tecnologia: remoção de material SRP (Subtrative Rapid Prototyping) e adição de material RP (SLS - Selective Laser Sintering e o FDM - Fused Deposition Modeling). Ademais, a tecnologia FDM foi inserida na fase de prototipagem do produto, por ser a tecnologia mais comumente utilizada na literatura e na maioria das empresas.

As Tabelas 6 e 7 apresentam o resultado da comparação entre os três processos de prototipagem pesquisados para os produtos 1 (protetor de cerdas) e 2 (escova dental). Ao analisar os protótipos do protetor de cerdas quanto à característica Tempo de execução, a tecnologia SRP apresentou uma redução de 50\% do tempo de execução do protótipo em relação às tecnologias SLS e FDM. Entretanto, o processo SRP apresentou alto custo de produção, não reprodução da geometria da cavidade do click, por representar um ângulo negativo para o processo de fresamento e não funcionamento do produto (ver Tabela 6).

No item Propósito do produto, as tecnologias SRP, SLS e FDM conseguiram formar os elementos que compunham o produto, entretanto, apenas o produto em processo SLS manteve a dobradiça e o click funcionando como o modelo projetado no CAD no primeiro movimento; os outros romperam.

Ao analisar os protótipos da escova dental quanto ao Propósito do produto e Dimensões, as tecnologias SRP, RP (SLS/FDM) apresentaram resultados semelhantes. Quanto ao item Tempo de execução, a tecnologia SRP apresentou uma redução de $70 \%$ do tempo de execução do protótipo e menor custo de 
Tabela 4. Matriz de priorização das características do produto 1 e das tecnologias

\begin{tabular}{|c|c|c|c|c|c|c|c|c|c|c|c|c|}
\hline \multicolumn{3}{|c|}{ Tecnologias } & \multicolumn{4}{|c|}{ SRP } & \multicolumn{6}{|c|}{$\mathbf{R P}$} \\
\hline & Características & 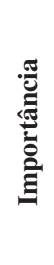 & 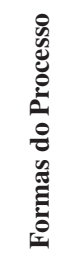 & 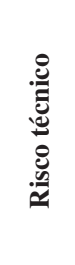 & 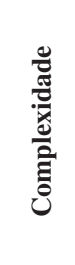 & 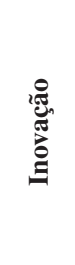 & 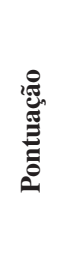 & 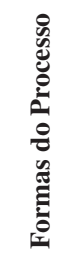 & 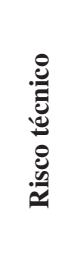 & 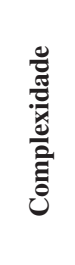 & 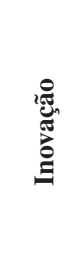 & 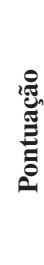 \\
\hline & & & $24 \%$ & $35 \%$ & $24 \%$ & $18 \%$ & & $17 \%$ & $20 \%$ & $30 \%$ & $33 \%$ & \\
\hline \multirow{8}{*}{ 营 } & Propósito & 9 & 1 & 1 & 0 & 9 & 20 & 9 & 9 & 9 & 9 & 81 \\
\hline & Material & 9 & 9 & 9 & 3 & 9 & 69 & 9 & 9 & 9 & 9 & 81 \\
\hline & Dimensão & 3 & 3 & 3 & 3 & 3 & 9 & 9 & 9 & 3 & 3 & 16 \\
\hline & Custo & 3 & 1 & 1 & 9 & 3 & 10 & 9 & 9 & 9 & 1 & 19 \\
\hline & Tempo & 3 & 9 & 9 & 9 & 1 & 23 & 1 & 1 & 9 & 3 & 13 \\
\hline & Detalhamento & 9 & 9 & 9 & 3 & 1 & 56 & 9 & 9 & 9 & 9 & 81 \\
\hline & $\begin{array}{l}\text { Acabamento } \\
\text { Sup. }\end{array}$ & 1 & 9 & 9 & 3 & 3 & 7 & 3 & 3 & 3 & 9 & 5 \\
\hline & Resultado & & \multicolumn{4}{|c|}{194} & \multicolumn{6}{|c|}{296} \\
\hline
\end{tabular}

Tabela 5. Matriz de priorização das características do produto 2 e das tecnologias.

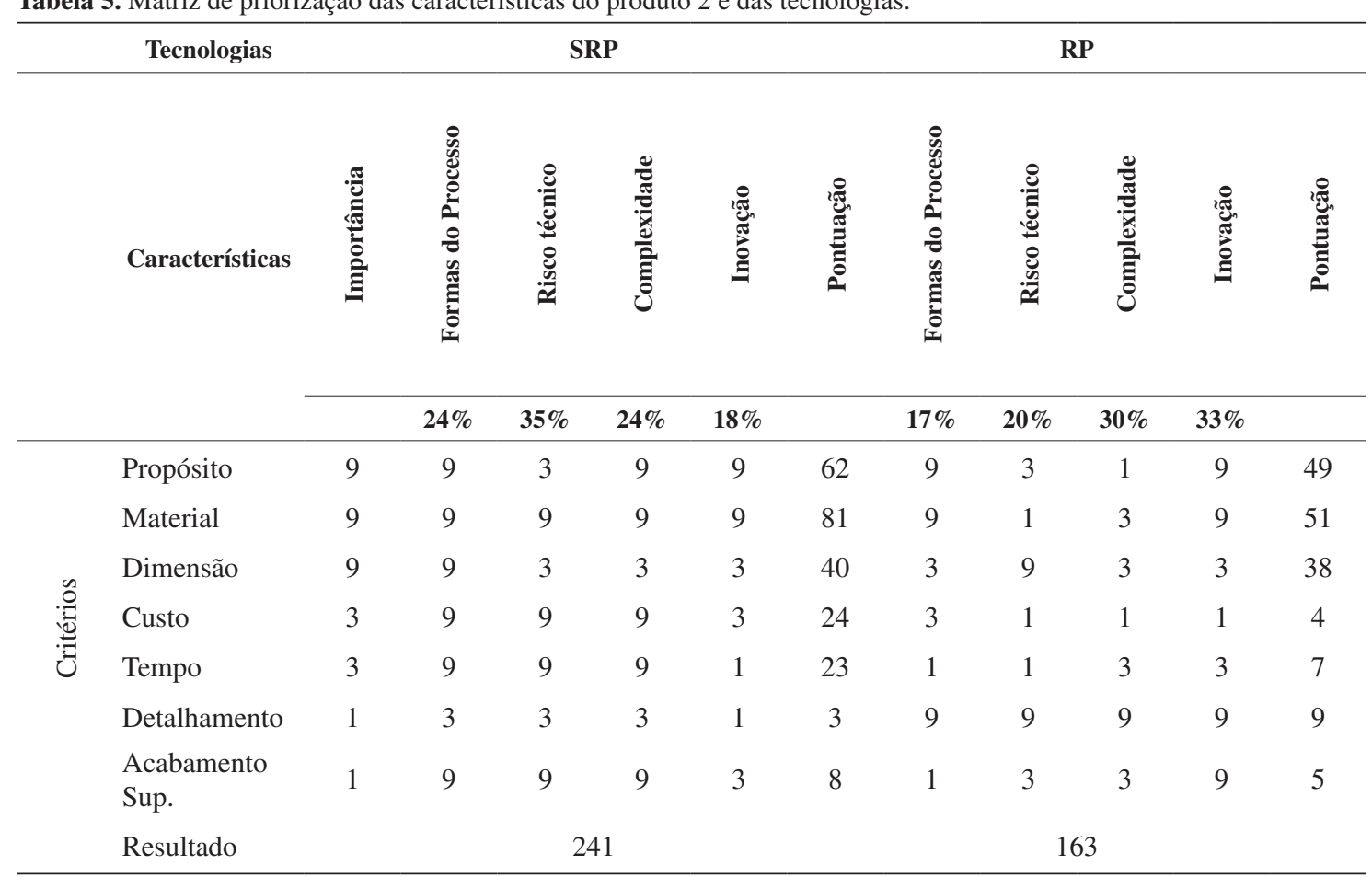

produção em relação à tecnologia RP (SLS/FDM). Quanto aos itens Detalhamento (furos e pegas) e Acabamento superficial, o protótipo produzido por SRP foi ótimo, não sendo necessário aplicar pós-acabamento na superfície final. Por sua vez, os protótipos das tecnologias SLS e FDM tiveram uma alteração dimensional $(1,90 \mathrm{~mm}$ e $1,60 \mathrm{~mm}$, respectivamente) e menor qualidade no acabamento (ver Tabela 7). Os resultados da prototipagem dos produtos corroboram com a análise de priorização dos critérios de decisão do método estabelecidos na Fase 2, destacando que a tecnologia SLS é mais 
Tabela 6. Comparação dos protótipos protetor de cerdas utilizando SRP e RP.

\begin{tabular}{|c|c|c|c|}
\hline \multirow{2}{*}{ Características } & \multirow{2}{*}{ SRP } & \multicolumn{2}{|c|}{$\mathbf{R P}$} \\
\hline & & SLS & FDM \\
\hline \multicolumn{4}{|l|}{ Visual dos protótipos } \\
\hline Propósito & $\begin{array}{l}\text { Não Funcionam } \\
\text { dobradiças e clicks }\end{array}$ & $\begin{array}{l}\text { Funciona } \\
\text { corretamente }\end{array}$ & $\begin{array}{l}\text { Não Funcionam } \\
\text { dobradiças e clicks }\end{array}$ \\
\hline Material & POM & PA & ABS \\
\hline Dimensões (mm) & $47,70 \times 37,50 \times 8,50 \mathrm{~mm}$ & $47,70 \times 37,50 \times 8,70 \mathrm{~mm}$ & $47,70 \times 37,50 \times 8,70 \mathrm{~mm}$ \\
\hline Custo & Alto & Baixo & Médio \\
\hline Tempo de Execução & 3 horas & 7 horas & 7 horas \\
\hline Detalhamento & Alto & Alto & Alto \\
\hline Acabamento Superficial & Alto & Médio & Médio \\
\hline
\end{tabular}

Tabela 7. Comparação dos protótipos escova dental utilizando SRP e RP.

\begin{tabular}{cccc}
\hline Características & SRP & \multicolumn{2}{c}{ RP } \\
\cline { 3 - 4 } Visual dos protótipos & FLS & FDM \\
Propósito & Funciona & Funciona & Funciona adequado \\
Material & POM & PA & ABS \\
Dimensões (mm) & $180 \times 12,40 \times 5,40 \mathrm{~mm}$ & $180 \times 12,40 \times 5,40 \mathrm{~mm}$ & $180 \times 12,40 \times 5,40 \mathrm{~mm}$ \\
Custo & Baixo & Médio & Alto \\
Tempo de Execução & 2 horas & 7 horas & 7 horas \\
Detalhamento & $1,80 \mathrm{~mm}$ (diâmetro) & $1,90 \mathrm{~mm}$ (diâmetro) & $1,60 \mathrm{~mm}$ (diâmetro) \\
& $2,50 \mathrm{~mm}$ (profundidade) & $2,50 \mathrm{~mm}$ (profundidade) & $2,50 \mathrm{~mm}$ (profundidade) \\
Acabamento Superficial & Alto & Baixo & Médio \\
\hline
\end{tabular}

adequada para o desenvolvimento do protótipo protetor de cerdas, e SRP para o protótipo escova dental.

\section{Conclusão}

Este artigo apresenta um método para tomada de decisão que auxilia a escolha da tecnologia de prototipagem rápida mais adequada na concepção de novos produtos. Foi realizada uma aplicação do método na concepção de dois produtos: um protetor de cerdas (produto composto leve) e uma escova dental (produto simples maciço), para avaliar o método proposto e validar os resultados obtidos.

Para deferir esse objetivo, o método proposto está organizado em três fases: a primeira fase estabelece a descrição das características e geometria do produto, bem como a intensidade de importância destas características pelo cliente. A segunda fase aplica o método de priorização das características (critérios) do produto e das tecnologias. E a terceira fase realiza a análise de tomada de decisão e prototipagem do produto com base na decisão da tecnologia mais adequada.

O método proposto apresenta duas contribuições relevantes para pesquisas em prototipagem rápida, sendo: (i) importância das características da tecnologia; (ii) priorização das características do produto ponderadas pelas características da tecnologia; e (iii) auxílio à tomada de decisão da tecnologia de prototipagem mais adequada.

Para o produto protetor de cerdas, a tecnologia RP (SLS) foi a mais bem-sucedida, tendo em vista o propósito do produto e suas funções de dobradiças e clicks. E para a escova dental, o melhor resultado alcançado foi da tecnologia SRP, pelo menor custo, menor tempo de execução, bom acabamento superficial, precisão dimensional e formação produto injetado nos furos de encerdamento.

Por fim, ressalta-se que a proposição de um método de simples utilização permite estabelecer qual tecnologia mais adequada para construir um 
protótipo com base na priorização das características relevantes do produto: propósito; custo; tempo; precisão dimensional e qualidade superficial, objetivando um protótipo mais eficiente. Esta proposta surgiu da constatação de lacuna nas aplicações de prototipagem rápida investigadas na literatura quanto ao auxílio científico na tomada de decisão.

Como pesquisas futuras, sugere-se analisar outras características de qualidade relevantes do produto, bem como o desenvolvimento de um programa computacional para o método de tomada de decisão proposto.

\section{Agradecimentos}

Os autores agradecem a Pontifícia Universidade Católica do Paraná pelo suporte financeiro, e aos revisores pela relevante leitura e contribuição.

\section{Referências}

Borille, A., Gomes, J., Meyer, R., \& Grote, K. (2010). Applying decision methods to select rapid prototyping technologies. Rapid Prototyping Journal, 16(1), 5062. http://dx.doi.org/10.1108/13552541011011712.

Campbell, R., De Beer, D., Barnard, L., Booysen, G., Truscott, M., Cain, R., Burton, M., Gyi, D., \& Hague, R. (2007). Design evolution through customer interaction with functional prototypes. Journal of Engineering Design, 18(6), 617-635. http://dx.doi. org/10.1080/09544820601178507.

Canciglieri, O. Jr., Sant'Anna, A. M. O., \& Machado, L. C. (2014). Decision making for prototyping of sustainable products. Chemical Engineering Transactions, 39, 529-534.

Evans, M. A., \& Campbell, R. I. (2003). A comparative evaluation of industrial design models produced using rapid prototyping. Rapid Prototyping Journal, 9(5), 344-354. http://dx.doi. org/10.1108/13552540310502248.

Ferreira, J. C., Santos, E., Madureira, H., \& Castro, J. (2006). Integration of VP/RP/RT/RE/RM for rapid product and process development. Rapid Prototyping Journal, 12(1), 18-25. http://dx.doi. org/10.1108/13552540610637237.

Hinloopen, E., Nijkamp, P., \& Rietveld, P. (2004). Inte- gration of ordinal and cardinal information in multicriteria ranking with imperfect compensation. European Journal of Operational Research, 158(2), 317-338. http://dx.doi.org/10.1016/j.ejor.2003.06.007.

Jain, S., Jiang, J., Huang, X., \& Stecandic, S. (2012). Modeling of fuel-cell-based power supply systems for grid interface. IEEE Transactions on Industry Applications, 48(4), 1142-1153. http://dx.doi.org/10.1109/ TIA.2012.2199454.

Machado, L. C., \& Sant'Anna, A. M. O. (2013). Uma visão dos processos de prototipagem rápida no desenvolvimento de produtos. Sodebras, 8(86), 86-93.

Min, H. (1994). International supplier selection: a multi-attribute utility approach. International Journal of Physical Distribution \& Logistics Management, 24(5), 2433. http://dx.doi.org/10.1108/09600039410064008.

Miranda, C. M. G., \& Almeida, A. T. (2004). Visão multicritério da avaliação de programas de pós-graduação pela Capes: o caso da área engenharia III baseado nos métodos ELECTRE II e MAUT. Gestão \& Produção, 11(1), 51-64. http://dx.doi.org/10.1590/S0104530X2004000100005.

Newcomer, J. L., Hoekstra, N. L., Kitto, K. L., \& Mckell, E. K. (2004). Using rapid prototyping to enhance manufacturing and plastics engineering technology. Journal of Engineering Technology, 21(1), 10-15.

Porto, A. J. V., Souza, M. C. F., Ravelli, C. A., \& Batocchio, A. (2002). Manufatura virtual: conceituação e desafios. Gestão \& Produção, 9(3), 297-312. http:// dx.doi.org/10.1590/S0104-530X2002000300007.

Sant'Anna, A. M. O. (2015). Framework of decision in data modeling for quality improvement. The TQM Journal, 27(1), 135-149. http://dx.doi.org/10.1108/ TQM-06-2013-066.

Sun, J., Chen, X., Liao, H., \& Xi, J. (2013). Template-based framework for nasal prosthesis. Rapid Prototyping Journal, 19(2), 68-76. http://dx.doi. org/10.1108/13552541311302914.

Volpato, N. (2007). Prototipagem rápida. São Paulo: Edgard Blutcher.

Warfel, T. Z. (2002). Prototyping: a practitioner's guide (3 ed.). New York: Rosenfeld LCC. 197 p.

Wohlers, T., \& Grimm, T. (2003). Is CNC machining really better than RP? Boston: Springer. 